\title{
Intoxicação aguda por triclorfon em caprinos tratados com a dose terapêutica ${ }^{1}$
}

\author{
Welber Daniel Zanetti Lopes ${ }^{2 *}$, Rafael Silveira Carvalho², Diogo dos Passos Gracioli², \\ Pedro Victor Oliveira ${ }^{2}$, Valdomiro Pereira ${ }^{2}$, Antônio Campanha Martinez ${ }^{2}$ \\ e Barbara Cristina Mazzucatto ${ }^{2}$
}

\begin{abstract}
Lopes W.D.Z., Carvalho R.S., Gracioli D.P., Oliveira P.V., Pereira V., Martinez A.C. \& Mazzucatto B.C. 2014. [Acute poisoning by trichlorfon in goats given a therapeutic dose.] Intoxicação aguda por triclorfon em caprinos tratados com a dose terapêutica. Pesquisa Veterinária Brasileira 34(2):114-118. Departamento de Medicina Veterinária, Universidade Estadual de Maringá, Campus Regional de Umuarama, Rodovia PR-489 no 1.400, Umuarama PR 87508-210, Brazil. E-mail: wdzlopes@hotmail.com

The present study describes an outbreak of trichlorfon poisoning in goats from the State University of Maringá, campus Umuarama/PR that received orally the therapeutic dose of the active ingredient in question $(100 \mathrm{mg} / \mathrm{kg})$. Fifty-three sheep had been treated with the same formulation/solution and no side effects were observed in any of these sheep medicated with triclorfon. But from 20 goats medicated with trichlorfon, eight goats showed, about 40 minutes after its administration, the classic clinical signs of ataxia, external lateral decubitus, drooling, tremors, constricted pupils, noisy dyspnea, involuntary urination and defecation, spastic paresis, bloat and tearing. Almost immediately after the detection of these signals, eight goats were medicated with $1 \%$ atropine sulfate $(0.5 \mathrm{mg} / \mathrm{kg}$ and fluid). Five of these goats received a second dose of atropine sulfate one hour after the first application because of some clinical signs such as muscle tremor still being present. Forty-eight to 72 hours after administration of trichlorfon, three of those five goats died. At necropsy we observed cyanotic mucous membranes, congestion of liver, spleen, kidneys and mesenteric vessels, filled gallbladder, emphysema, and reddish lungs. The results of this study call attention to the outbreak that occurred in adult goats in good clinical conditions and, above all, that they received the therapeutic dosage recommended on the manufacture label. This suggests a higher sensitivity of the species to the recommended dose of trichlorfon $(100 \mathrm{mg} / \mathrm{kg})$ for goats.
\end{abstract}

INDEX TERMS: Poisoning, insecticide, organophosphate, triclorfon, goats.

RESUMO.- 0 presente estudo descreve um surto de intoxicação por triclorfon em caprinos, que receberam pela via oral, a dosagem terapêutica do princípio ativo em questão $(100 \mathrm{mg} / \mathrm{kg})$, provenientes da Universidade Estadual de Maringá, campus de Umuarama, PR. Cinquenta e três ovinos foram tratados com a mesma formulação/solução e não se observou nenhum efeito colateral nos animais referente ao tratamento com triclorfon. Das 20 cabras medica-

\footnotetext{
${ }^{1}$ Recebido em 23 de setembro de 2013.

Aceito para publicação em 14 de novembro de 2013.

${ }^{2}$ Departamento de Medicina Veterinária, Universidade Estadual de Maringá (UEM), Campus de Umuarama, Rodovia PR-489 no 1400, Umuarama, PR 87508-210, Brasil. E-mail: wdzlopes@hotmail.com
}

das, cerca de 40 minutos após a administração do triclorfon, oito apresentaram os clássicos sinais clínicos de ataxia, decúbito externo-lateral, sialorreia, tremores, constrição das pupilas, dispneia com ruídos, micção e defecação involuntária, paresia espástica, timpanismo e lacrimejamento. Quase que imediatamente após a detecção destes sinais, as oito cabras foram medicadas com sulfato de atropina $1 \%$ $0,5 \mathrm{mg} / \mathrm{kg}$ mais fluidoterapia. Cinco destes animais tiveram de receber nova dosagem de sulfato de atropina uma hora após a primeira aplicação, em função de alguns sinais clínicos, como tremores musculares, ainda estarem presentes. De 48 a 72 horas após a administração do triclorfon, três destes cinco animais vieram a óbito. Na necropsia, foi possível observar mucosas cianóticas, congestão de fígado, 
baço e rins, vasos mesentéricos congestos, vesícula biliar repleta, enfisema pulmonar, parênquima pulmonar avermelhado. Os resultados encontrados neste trabalho chamam atenção que o surto aconteceu em cabras adultas, que apresentavam boas condições clínicas e acima de tudo, receberam a dosagem terapêutica recomendada em bula pelo fabricante. Talvez isso possa indicar alguma sensibilidade mais elevada desta espécie animal à dosagem recomendada em bula do triclorfon $(100 \mathrm{mg} / \mathrm{kg})$ para caprinos.

TERMOS DE INDEXAÇÃO: Intoxicação, inseticida, organofosforados, triclorfon, caprinos.

\section{INTRODUÇÃO}

Os nematódeos gastrintestinais são reconhecidos como um entrave na criação de pequenos ruminantes pela elevada perda econômica que estes parasitas podem causar nestes animais (Ejlertsen et al. 2006).

A fim de se minimizar os prejuízos ocasionados pelos helmintos, produtores e/ou proprietários destes animais, muitas vezes são obrigados a realizar a administração de formulações químicas de ação específica contra nematódeos. Dentre os diferentes grupos químicos de ação anti-helmíntica, indicado para pequenos ruminantes, têm-se os organofosforados, representado principalmente pelo triclorfon, que é administrado pela via oral. Este grupo químico foi originalmente desenvolvido para atuar sobre ectoparasitos. Entretanto, estudos posteriores e até mesmo recentes, demonstraram que estes fármacos, podem apresentar eficácia contra cepas de nematódeos de bovinos resistentes a elevadas concentrações de ivermectina (Lopes et al. 2009).

Princípios ativos pertencentes a este grupo químico são agentes anticolinesterásicos, possuem substâncias lipossolúveis de fácil absorção por toda superfície corpórea e, principalmente no trato gastrintestinal, pele, pulmão e olhos. Sua ação contra o parasita se da por meio da inibição irreversível da acetilcolinesterase, enzima que inativa a acetilcolina. 0 acúmulo de acetilcolina na fenda sináptica determina aumento no número de despolarizações, o que por sua vez ocasiona paralisia espástica e morte dos parasitas (Osweiler 1998, Barros et al. 2006).

Relatos de intoxicação por formulações pertencentes a este grupo químico vêm sendo descritos na literatura para bovinos, bubalinos, ovinos e até mesmo em seres humanos (Souza et al. 1996, Gava et al. 2001, Barbosa et al. 2003, Andrade \& Santarém 2006, Castro et al. 2007, Greco et al. 2009, Mendonça, et al. 2010, Oliveira-Filho et al. 2010, Dalto et al. 2011). Por este motivo, este grupo químico é considerado o mais tóxico para os animais vertebrados, quando estes por sua vez, acabam absorvendo os organofosforados em excesso, a ponto de desencadear sinais clínicos intoxicação nos animais. Sendo assim, a utilização de algumas formulações é realizada com diversas restrições, como: não ser administrada em fêmeas gestantes, não utilizar este grupo químico em fêmeas no terço final da gestação, em animais com menos de três meses de idade ou ainda em animais que estiverem consumindo uma alimentação rica em proteínas, de modo a não haver sobrecarga hepática (Barros \& Driemeier 2007, Radostitis et al. 2007).
Os sinais clínicos da intoxicação aguda nos hospedeiros vertebrados geralmente resultam da hiperestimulação do sistema parassimpático e incluem salivação, lacrimejamento, bradicardia, miose, tremores musculares, diarréia, fraqueza muscular, (Boermans et al. 1984, Maxie \& Youssef 2007, Zachary 2007).

Apesar do número de relatos de intoxicação de animais por organofosforados estar aumentando em função do incorreto uso na dosagem destes princípios ativos, ou serem administrados em categorias de animais não indicadas (animais jovens), ou mesmo, ainda, por questões de negligência de algumas pessoas, o presente trabalho tem como objetivo notificar a intoxicação aguda por triclorfon em caprinos adultos, clinicamente saudáveis, tratados pela via oral com a dosagem terapêutica do princípio ativo em questão $(100 \mathrm{mg} / \mathrm{kg})$. A justificativa para a utilização de $100 \mathrm{mg} / \mathrm{kg}$ de triclorfon por animal, se deve ao fato de que esta é a dose recomendada em bula da formulação em questão, bem como a utilizada em pesquisas científicas em camundongos (Salazar 2011) e também em ovinos (Cezar et al. 2011, Chagas et al. 2013).

\section{MATERIAL E MÉTODOS}

O surto ocorreu no mês de Agosto de 2012, no setor de ovinos e caprinos da fazenda experimental da Universidade Estadual de Maringá, Campus de Umuarama/PR. Este setor é composto por 130 ovinos, sendo quatro machos e 126 fêmeas (Texel e Santa Inês) entre dois e 36 meses de idade, e 20 cabras, sem raça definida, adultas não gestantes. Todos estes animais vivem em regime de pasto, durante o dia, se alimentando de gramíneas, e recebendo sal mineral e água ad libitum. A noite todos são presos em uma baia coletiva para os ovinos e outra para os caprinos, e durante este período, recebem capim picado e água ad libitum. Na manhã do dia seguinte, todos são soltos novamente nas pastagens. Esta rotina se repete todos os dias, com exceção de dois ovinos machos que são criados em baias e neste caso recebem volumoso/sal mineral e água ad libitum, além do concentrado em sua alimentação.

Contagens de ovos por grama de fezes (OPG), de acordo com a técnica descrita por Gordon \& Whitlock (1939), foram realizadas de todos os ovinos e caprinos, a fim de se constatar a carga parasitária dos animais para ovos do tipo estrongilídeos. Com base nestes resultados, optou-se em tratar 53 ovinos e as 20 cabras, todos criados a pasto.

Como tratamento, administrou-se o triclorfon, por via oral, na dose de $100 \mathrm{mg} / \mathrm{kg}$ em todos estes animais. A justificativa em se utilizar este medicamento, foi em função dos resultados prévios obtidos por Santos et al. (2012) no mesmo local onde este estudo foi conduzido. A dose máxima de medicamento foi de $5.000 \mathrm{mg} /$ $\mathrm{kg}$ por ovino e/ou cabra adulto. Dois dias anteriores ao tratamento, os 73 animais (53 ovinos e 20 cabras) foram pesados a fim de calcular a dose exata do medicamento. No dia do tratamento, os animais apresentavam um bom escore de condição corporal, além de estarem descansados.

Primeiramente os 53 ovinos foram tratados, e na sequência as 20 cabras. Após a administração da formulação, os animais foram mantidos nas baias coletivas por aproximadamente cinco horas recebendo capim picado, a fim de não serem submetidos a esforços físicos que pudessem alterar a sensibilidade dos animais a medicação administrada. A solução preparada e administrada foi a mesma utilizada nos ovinos e nos caprinos. 


\section{RESULTADOS}

Após a administração do triclorfon nos ovinos, não se observou nenhum sinal de anormalidade ou evento adverso nos animais referente à formulação em questão.

Por outro lado, cerca de 40 minutos após a administração do triclorfon nas cabras, oito das 20 apresentaram sinais clínicos sugestivos de intoxicação pelo princípio ativo supracitado. Estes oito animais começaram a apresentar ataxia, alguns ficaram em decúbito externo-lateral, apresentaram sialorreia (Fig.1), tremores, constrição das pupilas, dispneia com ruídos, micção e defecação involuntária (Fig.2), paresia espástica, timpanismo e lacrimejamento (Quadro 1). Logo após a detecção de tais sinais clínicos (cerca de 45 minutos após a administração do triclorfon e conseqüentemente cinco minutos após o início dos sinais de intoxicação), foi administrado, nos oito animais, via endovenosa sulfato de atropina $1 \%$ na dose de $0,5 \mathrm{mg} / \mathrm{kg}$ mais fluidoterapia como tratamento suporte. Cerca de 20 minutos após a administração do sulfato de atropina, os oito animais ficaram em estação novamente, entretanto, cinco ainda apresentavam tremores musculares e por este motivo receberam mais uma dose de sulfato de atropina

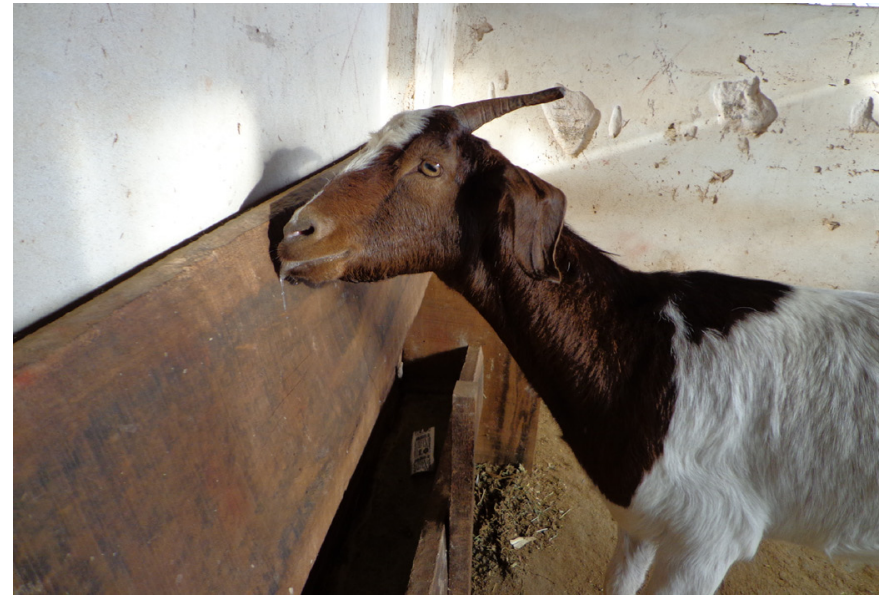

Fig.1. Cabra 93 com sialorreia e dificuldade de se manter em estação.
$(0,5 \mathrm{mg} / \mathrm{kg})$ uma hora após a administração da primeira dose (Fig.3). Estes animais permaneceram fechados até a resolução completa do problema, recebendo alimentação rica em fibras (volumoso) e água ad libitum.

Vinte e quatro horas após a administração da formulação, três animais, dos oito intoxicados, já se apresentavam em estação, se alimentando normalmente e por este motivo foram soltos a pastagem. Por outro lado, as cinco cabras que tiveram de receber outra dose de sulfato de atropina, ainda, voltaram a ficar em decúbito, apresentando apatia, anorexia (Fig.4) e por este motivo, continuaram recebendo tratamento suporte (fluidoterapia). Duas cabras (Cabras 91 e 110) e outra (Cabra 123) vieram a obtido 48 e 72 horas após a administração do triclorfon, respectivamente. Os dois animais que ficaram em decúbito, mas não vieram a óbito, conseguiram se recuperar dos sinais de apatia, anorexia e decúbito, no 4o dia após a administração do triclorfon, quando em seguida, foram soltas na pastagem juntamente com as demais cabras. Os três animais que vieram a óbito foram encaminhados para o departamento de Patologia Veterinária do campus desta mesma Universidade.

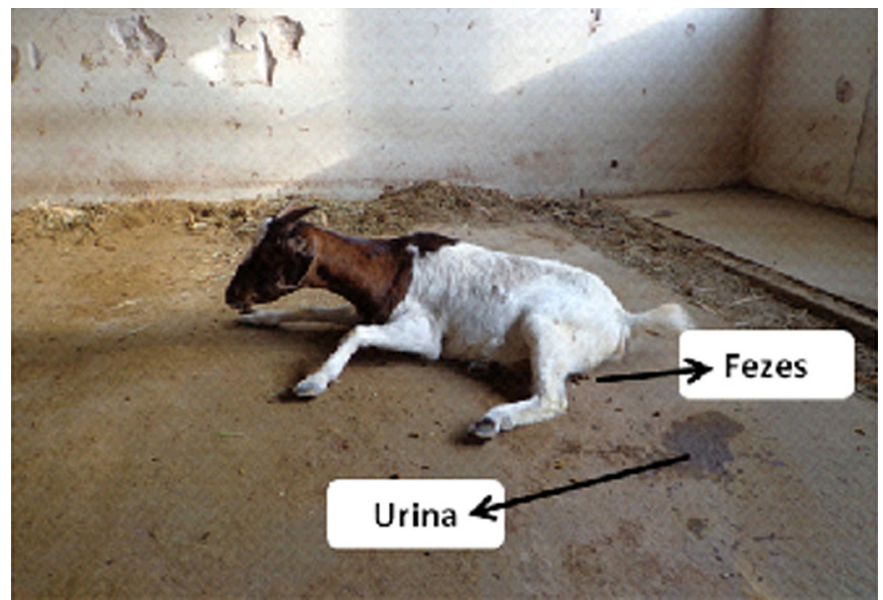

Fig.2. Cabra 93 demonstrando falta de equilíbrio, micção e defecação involuntária.

Quadro 1. Sinais clínicos apresentados pelas oito cabras intoxicadas por triclorfon, administrado via oral na dose terapêutica $(100 \mathrm{mg} / \mathrm{kg})$

\begin{tabular}{lcccccccc}
\hline \multicolumn{1}{c}{ Sinal clínico } & \multicolumn{7}{c}{ Cabra no. } \\
\cline { 2 - 9 } & 91 & 75 & 93 & 110 & 101 & 87 & 123 & 107 \\
\hline Decúbito externo-lateral & +++ & +++ & - & - & +++ & +++ & +++ & +++ \\
Ataxia & ++ & +++ & +++ & ++ & ++ & ++ & +++ & +++ \\
Constrição das pupilas & + & - & + & - & - & - & + & - \\
Dispneia com ruídos & - & - & +++ & ++ & - & - & ++ & ++ \\
Micção involuntária & + & + & + & + & - & - & + & - \\
Defecação involuntária & - & + & +++ & + & - & ++ & ++ & ++ \\
Sialorreia & ++ & ++ & + & ++ & +++ & ++ & +++ & +++ \\
Paresia espástica & ++ & ++ & ++ & ++ & + & ++ & +++ & ++ \\
Tremores musculares & +++ & +++ & + & ++ & +++ & ++ & +++ & +++ \\
Timpanismo & ++ & ++ & ++ & +++ & +++ & - & ++ & +++ \\
Lacrimejamento & + & - & - & - & - & - & + & - \\
Desfecho final & Morte & - & - & Morte & - & - & Morte & -
\end{tabular}

+++ Sinal acentuado; ++ sinal moderado; +sinal discreto; - ausência de sinal e/ou mortalidade. a morte do animal 48 horas após ${ }^{a}$ administração da formulação, ${ }^{b}$ morte 72 horas após a administração da formulação. 
Na necropsia das cabras, como achados macroscópicos foi possível observar mucosas cianóticas, congestão de fígado, baço e rins, vasos mesentéricos congestos, vesícula biliar repleta, enfisema pulmonar, parênquima pulmonar avermelhado (Quadro 2).

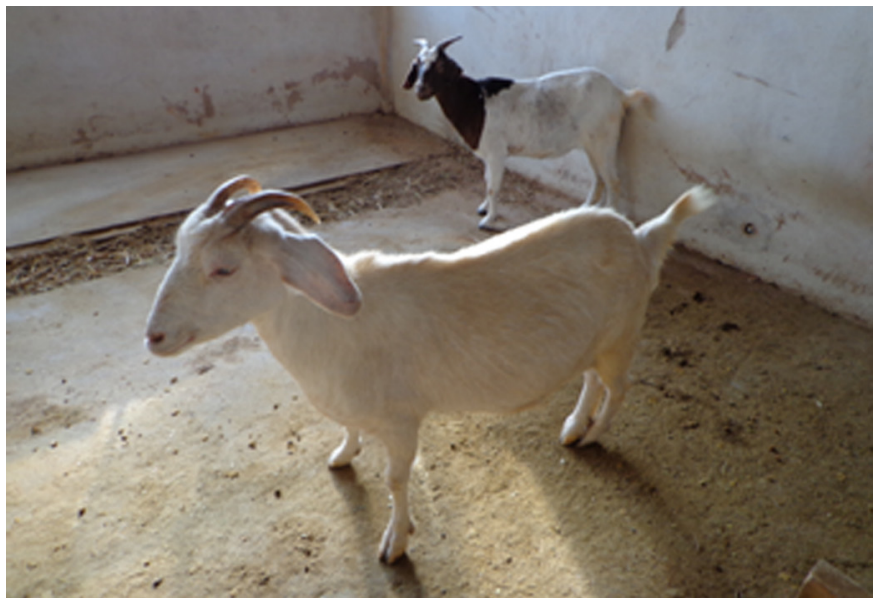

Fig.3. Cabra 123 (à frente) e Cabra 93 (atrás) cerca de dez minutos após a primeira administração de sulfato de atropina $1 \%$ $(0,5 \mathrm{mg} / \mathrm{kg})$.

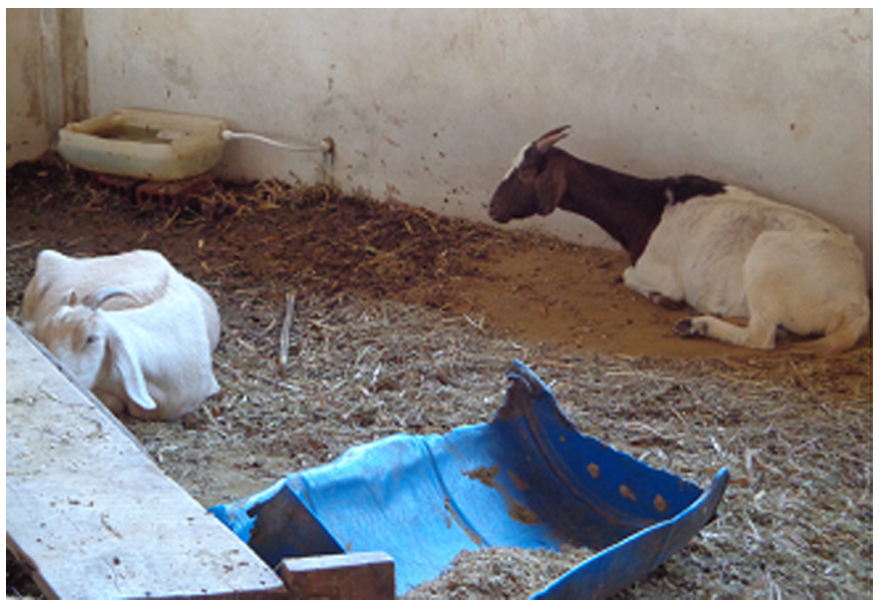

Fig.4. Cabra 123 (esquerda) e Cabra 93 (direita) 72 horas após a administração do triclorfon.

\section{DISCUSSÃO E CONCLUSÃO}

O diagnóstico de intoxicação por triclorfon foi realizado com base no histórico, epidemiologia, resposta dos animais ao tratamento com sulfato de atropina $1 \%$, sinais clínicos característicos e as alteração anatomopatológicas encontradas nas cabras que vieram a óbito. De acordo com Oliveira-Filho et al. (2010) e Dalto et al. (2011), o diagnóstico de intoxicação de animais aos organofosforados pode ser confirmado com base nos critérios pré-estabelecidos acima, além de exames toxicológicos, dependendo da situação ou falta de histórico do caso.

Os sinais clínicos de incoordenação motora, agressividade, sialorreia, tremores musculares, defecação involuntária, seguidos de morte em alguns casos, são clássicos de hiperestimulação do sistema nervoso autônomo (Pugh 1975, Khan 2001, Barros \& Driemeier 2007, Castro et al. 2007, Oliveira-Filho et al. 2008, Dalto et al. 2011).

De acordo com os relatos descritos na literatura (Pugh 1975, Khan 2001, Castro et al. 2007, Oliveira-Filho et al. 2010), a porcentagem média de mortalidade de animais intoxicados por este agente é em torno de $31 \%$ a $53,8 \%$ dos acometidos. Os resultados encontrados no presente trabalho estão de acordo com os descritos na literatura, uma vez que, $37,5 \%$ dos animais que foram intoxicados pelo triclorfon vieram a óbito em até 72 horas após a administração do princípio ativo em questão, mesmo recebendo duas doses de sulfato de atropina $1 \%$, mais fluidoterapia como tratamento suporte, quase que imediatamente após a detecção dos sinais clínicos sugestivos de intoxicação.

Em 2007, Castro e colaboradores na cidade de Paranoá-DF, relataram um caso de intoxicação de 11 de 23 bovinos adultos tratados com diazinon em concentração de cinco a seis vezes maiores que a recomendada pelo fabricante. Neste caso, a intoxicação foi confirmada pela pulverização de um bezerro com o restante do produto (calda), o qual demonstrou sinais clínicos após ter sido pulverizado com a mesma "calda". Ainda em relação a este caso, os animais intoxicados, foram submetidos a esforços físicos ao serem levados ("tocados") em um terreno bastante íngreme até a pastagem, logo após o tratamento, o que por sua vez aumentou a absorção do princípio ativo pela pele destes hospedeiros vertebrados.

Quadro 2. Achados anatomopatológicos das três cabras que vieram a óbito, provenientes da intoxicação por triclorfon administrado via oral na dose terapêutica $(100 \mathrm{mg} / \mathrm{kg})$

\begin{tabular}{lccc}
\hline Achados anatomopatológicos & \multicolumn{3}{c}{ Cabra no. } \\
\cline { 2 - 4 } & 91 & 110 & 123 \\
\hline Mucosa & Cianótica & Cianótica & Cianótica \\
Pulmão & Enfisema e parên- & Parênquima & Enfisema e parên- \\
& quima avermelhado & avermelhado & quima avermelhado \\
Fígado & Congestão & Congestão & NDN $^{\text {a }}$ \\
Vesícula biliar & Repleta & Repleta & Repleta \\
Baço & Congestão & Congestão & Congestão \\
Rúmen/Retículo/ & Hiperemia da & Hiperemia da & Hiperemia da \\
Omaso/Abomaso & mucosa & mucosa & mucosa \\
Mesentério & NDN & Vasos congestos & Vasos congestos \\
Rins & Congestão & Congestão & Congestão \\
Musculatura & NDN & NDN & NDN
\end{tabular}

${ }^{\mathrm{a}} \mathrm{NDN}=$ nada digno de nota. 
Mendonça et al. (2010) reportaram a morte de 11 de 25 vacas leiteiras intoxicadas, via pulverização, com uma formulação contendo organofosforado (diclorvós) mais piretróide (cipermetrina), em Cuiabá-MT. Os achados clínicos e anatomopatolológicos descritos por estes autores estão em concordância com os encontrados no presente estudo. Os autores não tinham conhecimento exato das condutas de aplicação (dosagem) da formulação em questão. 0 caso foi confirmado em função da detecção dos princípios ativos supracitados em alguns tecidos dos animais que vieram a óbito.

Neste mesmo ano (2010), Oliveira-Filho e colaboradores, notificaram no Rio Grande do Sul (cidade de São Sepe), intoxicações em uma propriedade de 49 animais intoxicados, sendo que destes, 20 vieram a óbito. Neste caso em específico, houve evidências de que a intoxicação foi proveniente da contaminação da água, por forato de uso agrícola, em função de uma empresa de aviação agrícola localizada pouco metros da propriedade acometida, havia lavado os equipamentos agrícolas contendo o agrotóxico em questão. A água proveniente dessa lavagem escorreu por gravidade e acumulou-se em poças na propriedade onde os bovinos acabaram ingerindo esta água contendo o produto e foram intoxicados.

0 último relato de intoxicação por organofosforados descrito na literatura aconteceu em bezerras da raça holandesa, com 15 dias de idade, tratadas, via pour-on, com ethion a $15 \%$ no Uruguai (Dalto et al. 2011). Os animais foram rapidamente medicados com sulfato de atropina $1 \%$, não havendo mortalidade destes. De acordo com os autores, o diagnóstico de intoxicação por organofosforado foi confirmado com base no histórico clínico dos animais, e na resposta ao tratamento dos animais ao sulfato de atropina $1 \%$.

0 que chama atenção é o fato de que, estas notificações de intoxicações descritas anteriormente aconteceram pelo uso de organofosforados em animais jovens, ou mesmo em animais adultos que receberam doses elevadas (superdosagem), ou, ainda, por negligência do ser humano, quando este submete animais a esforços físicos pré e/ou pós-administração de organofosforados, ou em casos quando este acaba contaminando a água ou alimento de animais com produtos pertencentes a este grupo químico. Entretanto, o surto agudo por triclorfon descrito no presente trabalho, ocorreu em cabras adultas, que apresentavam boas condições clínicas e acima de tudo, receberam a dosagem terapêutica recomendada em bula pelo fabricante. Talvez isso possa indicar alguma sensibilidade mais elevada desta espécie animal a dosagem recomendada de em bula do triclorfon $(100 \mathrm{mg} / \mathrm{kg})$ em caprinos, mesmo considerando todas as recomendações para o princípio ativo em questão.

\section{REFERÊNCIAS}

Andrade S.F. \& Santarém V.A. 2006. Manual de Terapêutica Veterinária. 2a ed. Roca, São Paulo. 697p.

Barbosa J.D., Alvares A.D. \& Barros C.S.L. 2003. Intoxicação por organofosforados em rebanho de bovinos. Anais Congresso Latino-Americano de Buiatria, Salvador, BA, p.21.

Barros C.S.L., Driemeier D., Dutra I.S. \& Lemos R.A.A. 2006. Doenças do Sistema Nervoso de Bovinos no Brasil: Coleção Vallée. AGNS, São Paulo. $207 p$

Barros C.S.L. \& Driemeier D. 2007. Intoxicação por organofosforados e carbamatos, p.80-85. In: Riet-Correa F., Schild A.L., Lemos R.A.A. \& Bor- ges J.R.J. (Eds), Doenças de Ruminantes e Eqüídeos. Vol.2. Pallotti, Santa Maria, RS.

Boermans H.J., Blak W.D., Chesney J., Robb R. \& Shewfelt W. 1984. Terbufos poisoning in dairy herd. Can. Vet. J. 25:335-338.

Castro M.B., Moscardini A.R.C., Reis J.L., Novaes E.P.F. \& Borges J.R.J. 2007. Intoxicação aguda por diazinon em bovinos. Ciência Rural 37:1498-1501.

Cezar A.S., Ribas H.O., Pivoto F.L., Sangione L.A. \& Vogel F.S.F. 2011. Combinação de drogas antiparasitárias como uma alternativa para o controle de nematódeos gastrintestinais multirresistentes em ovinos. Pesq. Vet. Bras. 31:151-157.

Chagas A.C., Katiki L.M., Silva I.C., Giglioti R., Esteves S.N., Oliveira M.C.S. \& Junior W.B. 2013. Haemonchus contortus: A multiple-resistant Brazilian isolate and the costs for its characterization and maintenance for research use. Parasitol. Int. 62:1-6.

Dalto A.G.C., Albornoz L., Gonzales P.C.S., Bitencourt A.P.G., Gomes D.C., Pedroso P.M.O. \& Bandarra P.M. 2011. Intoxicação por organofosforados em bezerros no Uruguai. Acta Scient. Vet. 39:983.

Ejlertsen M., Githigia S.M., Otineo R.O. \& Thamsborg S.M. 2006. Accuracy of an anemia scoring chart applied on goats in sub-humid Kenya and its potential for control of Haemonchus contortus infection. Vet. Parasitol. 141:291-301.

Gava A. 2001. Intoxicação por organofosforados e carbamatos, p.208-211. In: Riet-Correa F., Schild A.L., Lemos R.A.A. \& Méndez M.C. (Eds), Doenças de Ruminantes e Eqüinos. Vol.2. 2ª ed. Varela, São Paulo.

Gordon H.M. \& Whitlock H.V. 1939. A new technique for counting nematode eggs in sheep faeces. J. Counc. Scient. Indust. Res. 12:50-52.

Grecco F.B., Schild A.L., Soares M.P., Raffi M.B., Sallis E.S.V. \& Damés M.C. 2009. Intoxicação por organofosforados em búfalos (Bubalus bubalis) no Rio Grande do Sul. Pesq. Vet. Bras. 29:211-214.

Khan 0. 2001. Organophosphate poisoning in a group of replacement heifers and dry cows. Can. Vet. J. 42:561-563.

Lopes W.D.Z., Santos T.R., Borges F.A., Sakamoto C.A., Soares V.E., Costa G.H.N., Camargo G., Pulga M.E., Bhushan C. \& Costa A.J. 2009. Anthelmintic efficacy of oral trichlorfon solution against ivermectin resistant nematode strains in cattle. Vet. Parasitol. 166:98-102.

Maxie M.G. \& Youssef S. 2007. Nervous system, p.281-457. In. Maxie M.G. (Ed.), Jubb, Kennedy and Palmer's Pathology of Domestic Animals. Vol.1. $5^{\text {th }}$ ed. Saunders Elsevier, Philadelphia.

Mendonça F.S., Freitas A.H., Dória R.G.S., Camargo L.M. \& Neto J.E. 2010. Intoxicação por diclorvós e cipermetrina em bovinos em Mato Grosso: relato de caso. Ciênc. Anim. Bras. 11:743-749.

Oliveira-Filho J.C., Carmo P.M.S., Peirezan F., Tochetto C., Lucena R.B., Rissi D.R. \& Barros C.S.L. 2010. Intoxicação por organofosforado em bovinos no Rio Grande do Sul. Pesq. Vet. Bras. 30:803-806.

Osweiler G.D. 1998. Toxicologia Veterinária. Artes Médicas, Porto Alegre, RS. 526p.

Pugh W.S. 1975. An outbreak of organophosphate poisoning (Thimet) in cattle. Can. Vet. J. 16:56-58.

Radostitis O.M., Gay C.C., Hinchicliff K.W. \& Constable P.D. 2007. Veterinary Medicine: a textbook of the diseases of cattle, horses, sheep, pigs and goats. $10^{\text {th }}$ ed. Elsevier, Philadelphia. 2156p.

Salazar V.C.R. 2011. Investigação de mecanismos bioquímicos e fisiológicos em organismos expostos a anatoxina-a (s). Tese de Doutorado em Toxicologia e Análises Toxicológicas, Faculdade de Ciências Farmacêuticas da Universidade de São Paulo, São Paulo, SP. 187p.

Santos W.S., Carvalho R.S., Souza A.F.P., Oliveira P.V., Pereira V., Martinez A.C. \& Lopes W.D.Z. 2012. Eficácia anti-helmíntica de formulações comerciais, pertencentes a diferentes grupos químicos, contra nematódeos gastrintestinais de ovinos naturalmente infectados. Anais XVII Congresso Brasileiro de Parasitologia Veterinária, São Luis, Maranhão, p.26.

Souza M.V., Graça D.L. \& Cervo D. 1996. Neurotoxicidade tardia experimentalmente induzida por halaxon em ovinos. Arq. Bras. Med. Vet. Zootec. 48:275-286

Zachary J.F. 2007. Nervous System, p.914-915. In: McGavin M.D. \& Zachary J.F. (Eds), Pathologic Basis of Veterinary Disease. $4^{\text {th }}$ ed. Mosby Elsevier, St Louis. 